\title{
Development and application of Geospace Environment Simulator for the analysis of spacecraft- plasma interactions
}

\section{$\operatorname{AUTHOR}(S):$}

Usui, H; Miyake, Y; Okada, M; Omura, Y; Sugiyama, T; Murata, KT; Matsuoka, D; Ueda, HO

\section{CITATION:}

Usui, H ... [et al]. Development and application of Geospace Environment Simulator for the analysis of spacecraft-plasma interactions. IEEE TRANSACTIONS ON PLASMA SCIENCE 2006, 34(5): 2094-2102

\section{ISSUE DATE:}

2006-10

URL:

http://hdl.handle.net/2433/40008

\section{RIGHT:}

(c)2006 IEEE. Personal use of this material is permitted. However, permission to reprint/republish this material for advertising or promotional purposes or for creating new collective works for resale or redistribution to servers or lists, or to reuse any copyrighted component of this work in other works must be obtained from the IEEE. 


\title{
Development and Application of Geospace Environment Simulator for the Analysis of Spacecraft-Plasma Interactions
}

\author{
Hideyuki Usui, Yohei Miyake, Masaki Okada, Yoshiharu Omura, Tooru Sugiyama, Ken T. Murata, \\ Daisuke Matsuoka, and Hiroko O. Ueda
}

\begin{abstract}
Space development has been rapidly increasing, and a strong demand should arise regarding the understanding of the spacecraft-plasma interactions, which is one of the very important issues associated with the human activities in space. To evaluate the spacecraft-plasma interactions including plasma kinetics, transient process, and electromagnetic field variation, the authors have started to develop a numerical plasma chamber called Geospace Environment Simulator (GES) by making the most use of the conventional full particle-in-cell plasma simulations. For the development of a proto model of GES, the authors have used the Earth Simulator, which is one of the fastest supercomputers in the world. GES can be regarded as a numerical chamber in which space experiments can be virtually performed and temporal and spatial evolutions of spacecraft-plasma interactions can be analyzed. In this paper, the authors have briefly introduced GES in terms of its concept, modeling, and research targets. As one of the research topics of GES, the authors have investigated the impedance variation of electric field antenna onboard scientific satellites in the photoelectron environment in space. From the preliminary simulation results, the large change of reactance of the antenna impedance below the characteristic frequency corresponding to the local plasma frequency determined by the photoelectron density could be confirmed.
\end{abstract}

Index Terms-Numerical analysis, plasma waves, simulation software, space vehicle antennas.

\section{INTRODUCTION}

$\mathbf{T}$ HE SUCCESSFUL cooperation of the International Solar Terrestrial Program projects had yielded various space data simultaneously observed with much higher resolution than those of the previous missions (e.g., [1]). In parallel to these intensive observations with spacecraft, owing to exponential advancement in computer technology, numerical simulation

Manuscript received December 1, 2005; revised July 2, 2006. This work was supported in part by a joint research project in cooperation with the Earth Simulator entitled "Geospace Environment Simulator."

H. Usui, Y. Miyake, and Y. Omura are with the Research Institute for Sustainable Humanosphere, Kyoto University, Uji 611-0011, Japan (e-mail: usui@ rish.kyoto-u.ac.jp; y-miyake@ rish.kyoto-u.ac.jp; omura@ rish.kyoto-u.ac.jp).

M. Okada is with the National Institute of Polar Research, Itabashi 173-8515, Japan (e-mail: okada.masaki@nipr.ac.jp).

T. Sugiyama is with the Earth Simulator Center, Japan Marine Science and Technology Center, Kanazawa 236-0001, Japan (e-mail: tsugi@ jamstec.go.jp).

K. T. Murata and D. Matsuoka are with the Center for Information Technology, Ehime University, Matsuyama 790-8577, Japan (e-mail: murata@ cite.ehime-u.ac.jp; daisuke@ velvet.cs.ehime-u.ac.jp).

H. O. Ueda is with Japan Aerospace Exploration Agency Engineering Digital Innovation Center, Tsukuba 305-8505, Japan (e-mail: ueda.hiroko@ jaxa.jp).

Digital Object Identifier 10.1109/TPS.2006.883290 has progressed as a powerful tool for the detailed study of space plasma phenomena observed with spacecraft. Furthermore, simulations have potential possibility to predict plasma processes that have not been observed yet because of insufficient spatial and temporal resolutions of science instruments onboard spacecraft.

Meanwhile, space development has been rapidly increasing as shown in the construction of the International Space Station, In such a situation, a strong demand should arise regarding the understanding of the space environment around space structure, which is one of the very important issues associated with the human activities in space (e.g., [2]-[4]). One of the significant spacecraft-plasma interactions to be considered is spacecraft electrostatic (ES) charging because it is hazardous to onboard electronics. A spacecraft immersed in space attains some potential with respect to the surrounding space plasma because of accumulation of charged particles and other mechanisms such as photoemission and secondary electron emission. Since the 1960s, intensive studies on current collection to an electrical probe have been conducted by many researchers [5], [6]. The first observation of spacecraft charging at geosynchronous orbit was reported in [7]. Excellent reviews of the progress in the spacecraft charging field have been provided by Garrett [8], Garrett and Whittlesey [9], and Whipple [10]. Since ES charging is a common phenomenon for an object immersed in plasma, it has been widely explored from different perspectives by many communities including the dusty plasma [11]. Charging problem for electron-emitting surfaces has been also examined in many studies such as found in [12] and [13].

From the view point of numerical analysis of spacecraft charging, the National Aeronautics and Space Administration (NASA) developed NASA Charging Analyzer Program (NASCAP) as an engineering tool to determine the environmental effect on spacecraft surfaces and systems [14]. It has been developed mainly for obtaining the steady-state solution on the spacecraft charging in relatively short time for the engineering use because the obtained data are directly used for the spacecraft design. The latest version of NASCAP, called NASCAP2K, has the capability of particle-in-cell (PIC) [15] treatment for charged particles, and it computes only curlfree quasi-static field by solving Poisson's equation [16]. The European Space Agency started the Spacecraft Plasma Interaction System (SPIS) project in 2002, which aims at developing a software toolkit for spacecraft-plasma interactions and 
spacecraft charging modeling [17]. Although SPIS also has the capability of PIC simulation (e.g., [18] and [19]), it has been so far mainly used for obtaining the steady-state solution of spacecraft-plasma interaction problems. Furthermore, although SPIS has been designed such as to be easily extended for including new solvers, the current version of SPIS includes a Poisson's equation solver but no electromagnetic (EM) solver. Therefore, the spatial and temporal resolutions of SPIS simulations may be not enough for the analysis of the detailed microscopic and transient plasma processes in association with spacecraft-plasma interactions.

We, however, believe that transient variation caused by plasma kinetics is important because it may affect the steadystate solution on the spacecraft-plasma interactions. In addition, when we consider active plasma emission from spacecraft such as electric propulsion or plasma contactor, we need to examine the transient process of emitted plasma responses such as plasma acceleration in the process of ion beam neutralization by electrons. To do this, full PIC simulations are necessary, although they are very much costly because all the charged particles have to be treated as a large number of so-called superparticles in simulations.

In addition to the analysis of plasma transient process, disturbance of EM fields in the vicinity of spacecraft caused by the spacecraft-plasma interactions is of our great interest. When we focus on the characteristics of electric field antenna used for plasma wave observation in space, full Maxwell's equations have to be solved because the variation of magnetic field should be considered. In consideration of all the demands we raised above for the numerical studies on spacecraft-plasma interactions, we found that the existing numerical tools such as NASCAP and SPIS do not completely satisfy our demands and realized that we should develop our own simulation tool. To evaluate the spacecraft-plasma interactions including plasma kinetics, their transient process, and variation of EM field in self-consistent manner, we started to develop a numerical plasma chamber called Geospace Environment Simulator (GES) by making the most use of the conventional full PIC plasma simulation code such as KEMPO [20].

In Section II, we will describe GES concept and the research targets. In Section III, we will introduce one example of EM PIC simulations in association with spacecraft-plasma interaction. We particularly focus on the characteristics of electric field antenna onboard satellite in inhomogeneous plasma environment such as photoelectron sheath. Some of the fundamental results obtained in three-dimensional (3-D) EM PIC simulation are explained.

\section{GES}

\section{A. Concept and Method}

Fig. 1 shows the basic concept of GES. GES consists of two simulators in which we hire full PIC method [15]. One is the EM simulator, which enables us to investigate the EM interaction in association with the spacecraft-plasma environment. The analysis of electric field antenna characteristics in space plasma environment is one of the interesting research targets with the EM simulator. The other is the ES simulator, with

\section{Geospace Environment Simulator}

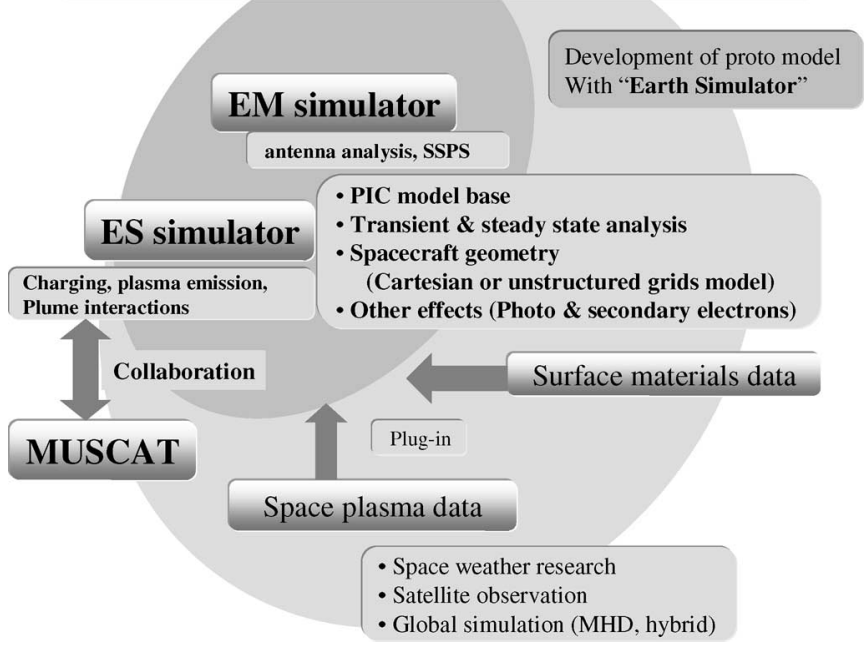

Fig. 1. Concept of GES.

which we can examine the ES phenomena such as spacecraft surface charging and plasma response in active plasma emission such as in electric propulsion and plasma-plume interaction. By making use of the ES simulator, the GES team has been assisting the development of the Multi-Utility Spacecraft Charging Analysis Tool (MUSCAT), which Japan Aerospace Exploration Agency started to develop as a numerical tool for obtaining the spacecraft charging at the steady state. The ES simulator of GES can be utilized for the quantitative validation of the MUSCAT results such as the current-voltage characteristics of a probe immersed in space plasma.

In the two simulators, we hire the standard PIC simulation method as described in [15] and [20]. At each time step, we update field values defined at grid points by solving Maxwell's equations with the standard finite-difference timedomain (FDTD) method [21]. In the ES simulator, we solve Poisson's equation for the ES field. The obtained fields are then applied to the equation of motion for updating the particles' velocity and positions. We use the Buneman-Boris method [20] for updating the particles' velocity. By introducing the PIC method, variation of fields defined at grid points and dynamics of plasma macroparticles located at arbitrary positions are updated in the self-consistent manner. In the current version of GES, spacecraft can be introduced in the simulation model as a solid body made of conducting or dielectric material, and its geometry is modeled by the Cartesian coordinate grids. To model more realistic geometry of a spacecraft, we plan to hire spacecraft modeling with unstructured grids. The characteristics of surface materials such as dielectric constant can be introduced in the ES simulation modeling, and they are considered in solving Poisson's equation to obtain the potential values on the spacecraft surface in using the capacity matrix method [22].

Electron emission from the spacecraft surface is also modeled with the standard method. By assuming a Maxwellian velocity distribution for electrons with some temperature independent of the background plasma, we calculate a flux value for 
the injection. The algorithm used to inject the electrons from the spacecraft surface is the same widely used in the literature [15]. In fact, numerical modeling of electron emission such as photoelectrons and secondary electrons from a conducting object is very difficult and has been investigated by many scientists (e.g., [12] and [23]). Regarding the background plasma environment, we will utilize the space plasma data obtained either by the space weather modeling, in situ satellite observations, or global magnetohydrodynamic simulations.

One of the significant features of GES is the highperformance computing for vector as well as parallel computer system. We originally started developing a proto model of GES by using the Earth Simulator [24]. The Earth Simulator, one of the fastest supercomputers in the world, consists of 640 nodes of vector-type supercomputer. Each node has 16-GB main memory shared with eight vector processors. The total size of the main memory is $10 \mathrm{~TB}$, and the maximum performance is 40 trillion floating point operations per second. For the most efficient performance of the Earth Simulator, each application is required to achieve the maximum optimization in vectorization and parallelization. In 2004, we started to develop the core engine of the EM simulator called NuSPACE, which contains the particle pusher and EM field solver in the domain decomposition manner. Owing to the intensive code tuning, we succeeded in achieving the maximum optimization of NuSPACE, the vectorization ratio of $99.7 \%$ and parallel efficiency of $100 \%$, on the Earth Simulator [25].

\section{B. Research Targets of GES}

GES can be considered as a numerical plasma chamber in which we can virtually perform space experiments and analyze the temporal and spatial evolutions of spacecraft-environment interactions. GES will be able to provide fundamental data regarding various engineering aspects such as the ES charging and EM interference of spacecraft immersed in space plasma. The obtained data will be useful and important in determining the design and the detailed specification of spacecraft and space system. Since there are several numerical tools available for the evaluation of steady-state solution of spacecraft charging, GES can be rather used for the analysis of transient plasma kinetics in the spacecraft-environment interactions and its effects on the spacecraft system.

In association of the spacecraft charging, plasma contamination due to thruster firings and ion engine operation can affect not only the spacecraft environment but also the spacecraft system in terms of sputtering and discharge. From a microscopic point of view, spacecraft interactions with the contaminated plasma environment, which consists of exhausted plasma plume, neutral gas, and ambient plasma, are to be quantitatively investigated. Particularly, to avoid the satellite anomaly caused by discharge, it is very important to evaluate the spacecraft surface charging, which is caused by ES interactions among plasma plume, spacecraft surface including solar paddle, and the ambient plasma (e.g., [2]). GES can contribute to the qualitative analysis of the transient plasma process as well as steady state of each interaction, which is closely associated with the spacecraft charging.
From the EM point of view, interaction of future Space Solar Power System (SSPS) [26] with the space environment is a good example for the GES. In SSPS, solar energy collected by a large number of solar cells attached to the satellite is converted to the microwave, and the energy is transmitted to a ground station or to another satellite by the microwave beam. To study the feasibility and the implications of SSPS, we started a research program of the microwave power transmission in the 1980 s by conducting rocket and ground experiments. The rocket experiments showed that ES waves were excited around the local plasma frequency in association with the microwave emission. Corresponding computer simulations revealed that the ES wave generation is due to the stimulated Raman scattering type of the nonlinear three-wave coupling process in the ionospheric plasma. The simulations also predict the electron heating associated with the ES wave excitation [27]. These simulation results are very interesting and important not only from the view point of plasma physics but also from the engineering side because they are useful in evaluating the threshold of the level of microwave power used for the transmission from SSPS to the ground. In this aspect, space simulation can be a powerful tool to obtain useful engineering information on how to minimize the undesirable effects to the space environment and to maximize the efficiency of power transmission in SSPS.

As mentioned earlier, the analysis of the antenna characteristics in space plasma environment is another important research issue for the GES because the quantitative understanding of antenna characteristics in plasma is required in the wave data calibration as well as in the antenna design for future missions. GES may be used not only for simulation of active experiments such as impedance measurements of complex array of antennas and probes but also for mutual impedance and relaxation sounder, all experiments which are designed for plasma diagnostics as they exist onboard different spacecraft.

In this paper, we particularly focus on the effects of photoelectron emission on the antenna characteristics. We started preliminary simulation study on the antenna impedance in the situation of photoelectron emission from the conducting surfaces of antenna and spacecraft. We will describe the 3-D EM PIC simulations on the antenna analysis in the next section.

\section{ANALysis of IMPEDANCE OF ELECTRIC FIELD Antenna in Photoelectron Environment in Space}

\section{A. Introduction}

For several decades, many scientists have investigated the antenna characteristics in plasma that are very complex because it is a dispersive and anisotropic medium. Therefore, in most of the previous theories, some assumptions were introduced in the antenna modeling. Under the cold plasma approximation, a formula of input impedance of a short dipole antenna was theoretically derived with the induced electromotive force (emf) method as described in [28]. Theoretical analyses of input impedance for dipole antenna with warm plasma approximation were also done for some limited models (e.g., [29]-[31]). Natural thermal noise in plasma waves can be evaluated by the using the kinetic impedance [32]. In these works, triangular current distribution is assumed on the antenna surface because 
the wavelength applicable in the theories is much larger than the antenna length. However, it is shown that the triangular approximation is not always valid particularly near the plasma frequency even in the case of long wavelength [33]. To determine arbitrary current distribution on the antenna surface, several attempts have been proposed such as a transmission line theory [34], which is applicable to any antenna length. Another important point to be considered in the antenna analysis in plasma is the inhomogeneous plasma distribution around the antenna. In many previous works, antenna is assumed to be a thin conductive wire, totally transparent to the fluid plasma medium. In fact, however, antenna surface is a solid body, and plasma particles that impinge the surface are absorbed at the solid surface and contribute to the charging. In this situation, an electron-free layer called ion sheath is formed around the antenna surface. By introducing the electron-free layer around the antenna surface, antenna impedance has been examined by many works (e.g., [35]), and it has been pointed out that the ion sheath has prominent contribution on the antenna impedance. It was also shown that impedance variation at the plasma frequency is significantly different from the impedance obtained in the transparent antenna model [36]. In these works, the conventional induced emf method that assumes a fixed model of current distribution has been hired, which is not applicable to the general antenna modeling including complex geometry, plasma kinetics, and plasma inhomogeneity near the antenna surface. To conquer this difficulty, the usage of the surfacecharge distribution (SCD) method has been proposed, in which all boundary surfaces can be considered including antenna geometry and ion sheath interfaces [37]. In this method, the ion sheath interface is given as a parameter. The antenna analysis with the SCD method has been successfully applied to the interpretation of impedance measurements onboard spacecraft such as the CLUSTER satellite [38].

Meanwhile, recent progress of computer facilities enables us to analyze the antenna properties in vacuum with the FDTD method, which solves the Maxwell's equations with spatial and temporal grid points [19]. The merit of FDTD simulation is that quantitative analysis of realistic antenna geometry is possible without assuming any current distribution on antenna surface (e.g., [39] and [40]). By using the FDTD method with the plasma fluid approximation, we can analyze the antenna impedance in plasmas (e.g., [41]). However, to include the plasma kinetic effects such as electron temperature and plasma sheath around the antenna, we need to treat the plasma as particles in the FDTD simulations. For this aim, we applied for the first time the PIC method to the conventional FDTD field solving simulation for the antenna analysis. Regarding the antenna model, we introduce a solid conductor for antenna surface, inside of which the electric field is zero. Plasma particles impinging into the antenna surface are accumulated and contribute to the spacecraft charging. With this antenna modeling, plasma-antenna interactions can be self-consistently simulated, and inhomogeneous plasma distribution such as ion sheath around the antenna is numerically realized with no assumption of current distribution or plasma-sheath interface.

In the following section, we will report some of the results regarding the antenna impedance obtained in the PIC simulations.

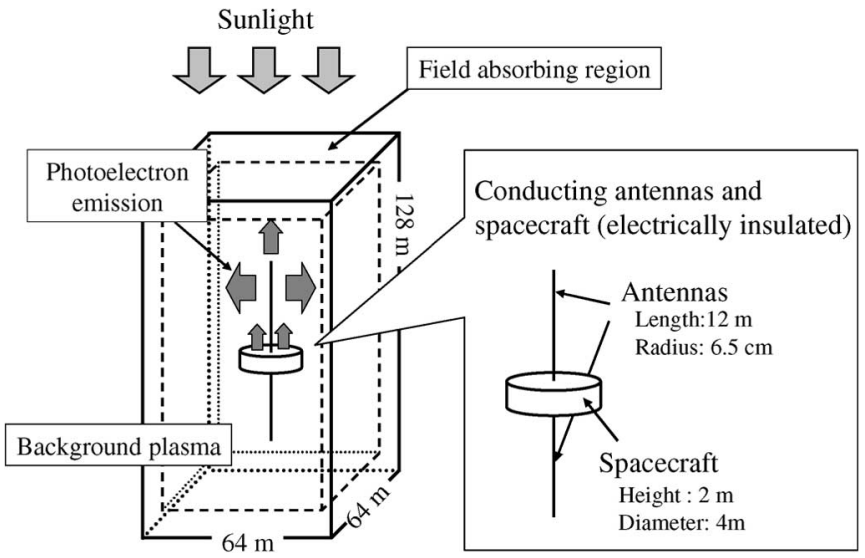

Fig.2. 3-D simulation model for the antenna analysis in the photoelectron environment.

We particularly modeled a situation of photoelectron emission from the antenna surface and investigated the dependence of antenna impedance on the photoelectron density in the frequency domain. In addition to the photoelectron environment, we included spacecraft geometry in the simulation model. In the future mission currently being planned by the Japanese community of solar terrestrial physics, spin-axial electric field antennas are planned to be implemented to realize 3-D measurements of electric field. To retain the attitude stability and lightweight, the spin-axial antennas need to be well short in comparison to the conventional wire antenna. Prior to the design of the short antenna, we started to examine the antenna characteristics by considering spacecraft body effects. The simulation model and some fundamental results on the antenna impedance are described in the following sections.

\section{B. Simulation Model}

By modifying the 3-D EM PIC simulation code called KEMPO (e.g., [20]), we developed a plasma simulation tool for the analysis of the antenna-plasma interactions. The modified code can be considered as a proto model of the GES EM simulator. To model the antenna in the PIC simulation, we made two major modifications in the code. One is the introduction of conductive solid bodies as inner boundaries in the simulation space, and the other is the electron emission from the bodies, which simulates photoelectron emission.

We show the 3-D simulation model in Fig. 2. We set a spacecraft body and two monopole antennas in the center, each of which is attached to the top and bottom surfaces of the spacecraft, respectively. In the current simulation as shown in Fig. 2, the antenna length is set to be much shorter than the conventional ones, and the dimensions of the spacecraft cannot be ignored in comparison with the antenna length. We assume that the antennas and spacecraft body are pure conductors. Therefore, the electric field in the bodies is zero. To obtain the equipotential solution for the conducting bodies, we redistribute the surface charge by using capacity matrix method (e.g., [22]) and correct the ES field by solving Poisson's equation in consideration with the modified surface charge. This modification of the electric field at the conducting surface should be done every 
time step. The joints between the antennas and the spacecraft body are assumed to be electrically insulated because of the connection with high impedance.

As another modification in the simulation code, we added a function that handles electron emission from the inner boundaries. In the current model, the inner boundaries are conducting bodies representing the antennas and spacecraft. As stated in Section II, we use the standard algorithm for the electron injection from the conducting bodies [15]. By using this function of electron injection, we simulate photoelectron emission from the sunlit surfaces of the spacecraft body and the antenna attached to the top surface of the spacecraft, which is illustrated in Fig. 2. In the present analysis, we assumed that only monopole antenna and one side of spacecraft surface are sunlit as the simplest case. The emitted photoelectrons are assumed to have a Maxwellian velocity distribution with $3.9 \times 10^{2} \mathrm{eV}$. To emphasize the effect of the photoelectrons to the antenna environment, the current density at the top surface of the spacecraft is assumed as $6.28 \times 10^{-2} \mathrm{~A} / \mathrm{m}^{2}$, which is much larger than the real parameter. Since the direction of the sunlight is set to be parallel to the antenna length, the amount of photoelectrons at the antenna surface is much less than that at the top surface of the spacecraft. Therefore, we assumed that the current density at the antenna surface is one-fifth of the value for the top surface of the spacecraft. The net charge on the conductive surface is counted at each time step in consideration of the electron charges that are emitted as photoelectrons and collected as return electrons, as well as the thermal background electrons impinging at the surface. By using the net charge and the charge of the background plasma, we solve Poisson's equation to obtain the potential values and the electric field at each grid point as stated above. Then, the floating potential of the antennas and the spacecraft body can be obtained in the self-consistent manner.

In this paper, we combined two simulation runs for the antenna analysis. The parameters used in the simulations are listed in Table I. First, we perform an ES simulation that focuses on the creation of plasma environment around the spacecraft. Initially, the simulation region is uniformly filled with the background electrons with the Debye length of $4.5 \mathrm{~m}$. Since we are interested in the frequency range corresponding to the electron dynamics, we ignore the ion dynamics by hiring immobile background ions for the charge neutralization in the simulation space. In the current mode of simulation, we solve Poisson's equation for the ES field and equations of motion for the electrons with the standard PIC method [15]. We perform the simulation with sufficient time steps, so that the floating potentials of the sunlit antenna and the spacecraft body reach the steady-state values. At this stage, the amounts of escaping and impinging electron currents come to be balanced together with the incoming background thermal electrons at each conducting body.

After obtaining the plasma environment around the spacecraft and antenna with the above ES simulation, we move to the EM simulation for antenna analysis, where we solve Maxwell's equations for the EM field and equations of motion for the electrons with the usual PIC method. In the EM simulation, we use the photoelectron sheath environment created in the ES simulation as the initial plasma environment. To obtain
TABLE I

PARAMETERS USED IN THE SimUlation

\begin{tabular}{|c|c|c|c|}
\hline \multicolumn{4}{|l|}{ Background electrons } \\
\hline \multicolumn{2}{|c|}{ Density } & $2.82 \times 10^{9}$ & {$\left[/ \mathrm{m}^{3}\right]$} \\
\hline \multicolumn{2}{|l|}{ Temperature } & 1.55 & {$[\mathrm{keV}]$} \\
\hline \multicolumn{2}{|l|}{ Debye length } & 4.5 & {$[\mathrm{~m}]$} \\
\hline \multicolumn{4}{|l|}{ Photoelectrons } \\
\hline \multirow[t]{2}{*}{ Current density } & (top surface of the spacecraft) & $6.28 \times 10^{-2}$ & {$\left[\mathrm{~A} / \mathrm{m}^{2}\right]$} \\
\hline & (sunlit antenna) & $1.256 \times 10^{-2}$ & {$\left[\mathrm{~A} / \mathrm{m}^{2}\right]$} \\
\hline \multicolumn{2}{|l|}{ Temperature } & 388 & {$[\mathrm{eV}]$} \\
\hline \multicolumn{4}{|l|}{ System parameters } \\
\hline \multirow{4}{*}{$\begin{array}{l}\text { Unit grid length } \\
\text { Number of grids }\end{array}$} & $: \mathrm{dr}$ & 0.5 & \multirow[t]{4}{*}[\mathrm{m}]{} \\
\hline & $: \mathrm{Nx}$ & 128 & \\
\hline & :Ny & 128 & \\
\hline & $: \mathrm{Nz}$ & 256 & \\
\hline Time step width & :dt & $1.67 \times 10^{-9}$ & {$[\mathrm{~s}]$} \\
\hline \multicolumn{2}{|c|}{ Number of superparticle per grid } & 8 & [/cell] \\
\hline
\end{tabular}

the input impedance of the antenna, we adopted the delta-gap feeding method, which has been widely used in the antenna analysis in free space [40]. In this method, we treat the antenna as a transmitting one and provide voltage $V_{i}$ of a Gaussian-type pulse at the power-feeding point, which corresponds to the joint between the sunlit antenna and the top surface of the spacecraft body. We then obtain the amount of current flow $I_{i}$ induced at the feeding point from Faraday's law by making use of the four components of rotational magnetic field around the feeding point. The antenna input impedance $Z$ is first calculated as the ratio $V_{i} / I_{i}$ in the time domain and then transformed to a frequency domain by taking Fourier transformation of $Z$ to obtain the real and imaginary parts of impedance. We set field absorbing region at the outer boundaries of the simulation box to avoid the wave field reflection from the boundary. To maintain the photoelectron environment in the EM simulation, we continue the electron emission and correction of the surface charge on the conducting bodies.

\section{Simulation Results}

1) Plasma Environment With Photoelectron Emission: By performing the ES simulation with the model described above, we created the inhomogeneous plasma distribution in the vicinity of the spacecraft system in a situation of photoelectron emission from the conducting surfaces. Fig. 3 shows a contour map of electron density around the antennas and spacecraft body when the floating potentials of the sunlit antenna and the spacecraft body reach the steady state. The contour map is obtained in the $x z$ plane, which includes the center of the antenna and spacecraft. The amount of the electron density is plotted in accordance with the gray scale in Fig. 3, with the highest value in white and the lowest in black. As mentioned above, we hired a model in which photoelectrons are emitted from the upper antenna and the top surface of spacecraft body. As clearly shown in Fig. 3, a photoelectron sheath with high electron density shown in white is created around the upper antenna and the upper surface of the spacecraft body. Since we assumed that the photoelectron flux from the top surface of the spacecraft body is five times larger than the flux from the antenna surface, it is shown that the photoelectron density 


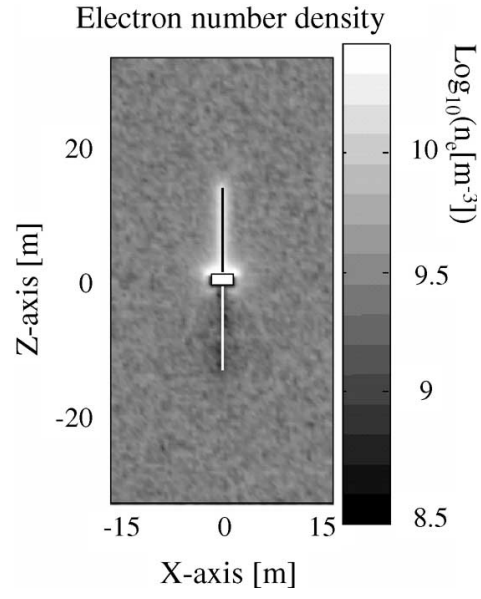

Fig. 3. Contour map of electron density around the spacecraft and antenna.

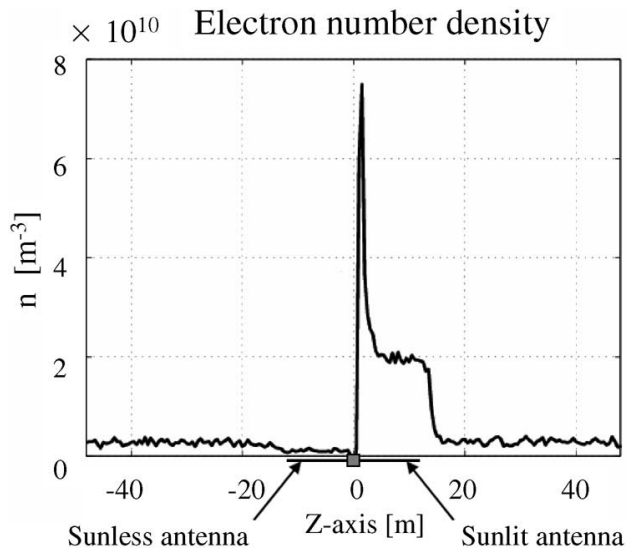

Fig.4. Density variation along the antenna length measured at the antenna surface.

around the upper surface of spacecraft body is much higher than the density around the upper antenna. Meanwhile, the sunless antenna, the lower one in Fig. 3, is negatively charged because of background electrons impinging to the conducting surface. Then, an ion sheath, which is an electron-sparse region, seems to be created around the sunless antenna. The density variation in the ion sheath is hard to be recognized in the contour map.

To ensure the electron distribution at the conducting surfaces, we plot the density values measured at one grid above the conduction surface along the antenna direction in Fig. 4. As shown in the previous contour map, the maximum electron density, which is approximately 25 times the average background density, is found at the right side of the spacecraft, which corresponds to the sunlit surface. Around the sunlit antenna that is attached at the right side of the spacecraft, photoelectron sheath with density of $2 \times 10^{10} \mathrm{~m}^{-3}$ is confirmed in Fig. 4. In contrast with the sunlit antenna surface, electron density is less than that of the background around the sunless antenna. In fact, at the last step of the ES simulation, the sunless antenna has not reached the steady state in terms of the floating potential. It seems that the potential of the sunless antenna is still decreasing and takes a longer time to reach the steady value of the floating potential. In the current simulation in which immobile ions are introduced for the charge neutralization, the steady state for the sunless an- tenna can be obtained only when the electron thermal flux at the antenna surface becomes completely zero. However, the zero electron flux is almost impossible because there always exist high-energy electrons that can pass the potential barrier created by the negatively charged antenna and can impinge into the antenna body. Therefore, it takes a very long time to reach the quasi-steady state for the sunless antenna. On the other hand, for the sunlit antenna, the current of emitted photoelectrons can be balanced with that of the impinging background electrons plus the returning photoelectrons. Since the photoelectron flux is much larger than the thermal electrons in the present model, the characteristic timescale for the photoelectron response is relatively short.

In general, the electron density at the steady state depends on the ion dynamics. From this point of view, the electron density and the associated antenna floating potential obtained in the immobile ion model are not rigorous for the steady state. In the vicinity of the sunlit antenna, however, the background density is much lower than the photoelectron density. Therefore, even if we assume the mobile ion, the photoelectron current becomes dominant, and the contribution of ion flux to the floating potential will be very small. Considering the abovedescribed situation, the electron density obtained in the current simulation will be less affected by the ion dynamics, and it can be considered as that for the quasi-steady state. Therefore, the obtained photoelectron environment can be used for the analysis of the antenna impedance described in the next section.

2) Photoelectron Effects on the Reactance of the Antenna Impedance: By using the inhomogeneous plasma environment self-consistently obtained with the ES simulation described above, we performed EM simulation for the analysis of the antenna impedance. In the EM simulation, we initially loaded the electrons that are saved at the last step of the previous ES simulation and created the initial electron distribution in the same 3-D simulation box as used in the ES simulation. In this paper, our focus is on the variation of the reactance of the antenna impedance in the photoelectron sheath environment. Therefore, we analyze the impedance of the sunlit antenna. In the EM simulation as described in the previous section, we used the delta-gap method to obtain the data of induced current at the power-feeding point, which is the junction between the sunlit antenna and the top surface of the spacecraft. By taking the Fourier transformation of the ratio between the voltage we provided at the feeding point and the induced current, we can evaluate the antenna input impedance in frequency domain.

Fig. 5 shows the reactance, which is the imaginary part of the antenna impedance, as a function of frequency. The solid and dash-dotted lines correspond to the reactance of the sunlit antenna and of the vacuum case, respectively. As shown clearly, there is a large difference between the two curves particularly at the low frequency range. Considering that the impedance variation can be seen mostly at the plasma frequency according to the past studies, this difference of reactance observed in the broadband of frequency region cannot be simply explained with the conventional theory. This drastic variation of reactance could be rather caused by the enhancement of electron density by the photoelectron sheath. To examine the effect of the photoelectrons, we define the following characteristic frequency 


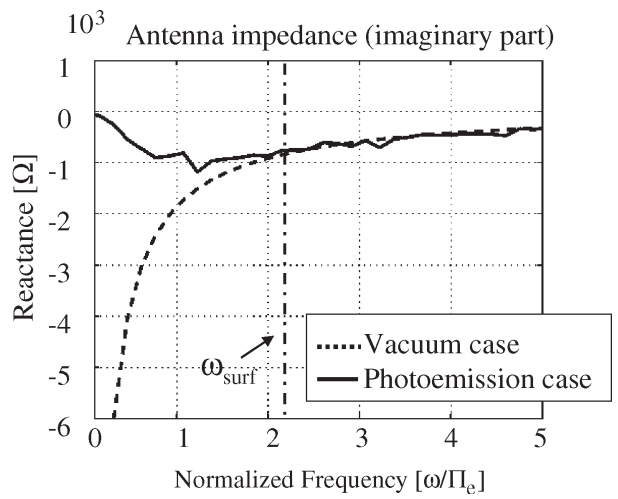

Fig. 5. Reactance values (the imaginary part of impedance) versus frequency.

that represents the plasma frequency in consideration of the average density of electron $n_{\text {surf }}$ at the antenna surface:

$$
\omega_{\text {surf }}=\sqrt{\frac{n_{\text {surf }} e^{2}}{m_{e} \varepsilon_{0}}}
$$

where $e, m_{e}$, and $\varepsilon_{0}$ denote the electron charge, mass, and dielectric constant for vacuum, respectively. When we plot $\omega_{\text {surf }}$ in Fig. 5, it is clearly shown that there is a change of the reactance below $\omega_{\text {surf }}$, while there is a little difference above $\omega_{\text {surf }}$ from the curve for the vacuum. We will discuss the result in the following.

When a dipole antenna interacts with the ES wave such as the Langmuir wave, the antenna shows a frequency resonance at the plasma frequency determined by the background density. As examined in many past works (e.g., [28]), it is shown that the resistance, the real part of the impedance, has a peak value, and the reactance, the imaginary part, shows a drastic change both at the plasma frequency. The impedance modification by ES waves depends on the Debye length and its relative ratio to the antenna length, which is discussed in the latest work with the CLUSTER antenna modeling [38]. In the current simulation, the Debye length of the background plasma is $4.5 \mathrm{~m}$, while the entire length of the antenna is $24 \mathrm{~m}$. In this situation, the signature of the resonance around the plasma frequency of the background plasma is rather difficult to be observed because it is generally known that it appears for antenna lengths around 8-10 m Debye lengths. Meanwhile, in the photoelectron sheath environment, the electron density is much higher, and the corresponding local Debye length is shorter. In such a situation, we can expect that a signature of impedance resonance is found with a large change of the reactance in the impedance plot around the local plasma frequency determined by the photoelectron density. As shown in Fig. 5, however, a large overshoot like change of the reactance is not observed at the plasma frequency of the photoelectrons. Instead, we observed large modification of the reactance in a broad frequency range particularly below $\omega_{\text {surf }}$.

The discrepancy between the profile of the reactance obtained in the current result and those in the past works can be caused by the antenna modeling, particularly the treatment of the electron particles impinging to the antenna solid surface. In the present model, we can handle the effect of the electron conduction current induced by actual motion of photoelectrons impinging the conducting solid surfaces of the antenna. Although some past works (e.g., [36]) focused on the effects of the plasma conduction current in the solid antenna model, the effect in the photoelectron environment has been little discussed so far. In the aspect of electric circuit, we can assume a resistance with a finite value that controls the electron conduction current between the antenna and the spacecraft surface. This resistance is different from the radiation resistance. At the low-frequency region, this additional resistance $R_{c}$ can be placed in parallel to the capacitance $C$ assumed between the antenna and the spacecraft [42]. Then, the reactance $X$ can be given as $-\omega C R_{c}^{2} /(1+$ $\left.\left(\omega C R_{c}\right)^{2}\right)$, where $\omega$ denotes frequency. By using this formula, it is easily shown that $X$ tends to be zero when $\omega$ approaches to zero. This tendency of the reactance modification qualitatively agrees with the profile shown in Fig. 5.

By using the above equivalent circuit, the resistance $R$, which is the real part of the impedance, is given as $R_{c} /(1+$ $\left.\left(\omega C R_{c}\right)^{2}\right)$, which gradually increases as the frequency approaches to zero in the frequency region below $\omega_{\text {surf }}$. Although not displayed, this tendency is qualitatively similar to our simulation result. However, this resistance variation is different from the result obtained in the previous work with the kinetic theory [36]. To examine the difference in detail, we have been working on the further quantitative analysis of the profiles of the resistance and reactance. The detail including the comparison with the PIC simulation results will be reported in another article in the near future.

In the present antenna model, we adopted unrealistic values of energy and flux for the electron emission from the conducting bodies. As stated earlier, this attempt was made to emphasize the effect of the photoelectron environment on the antenna characteristics by distinguishing $\omega_{\text {surf }}$ from the background plasma frequency in the impedance profile shown in Fig. 5, in which $\omega_{\text {surf }}$ becomes almost twice the background plasma frequency. In reality, the density of the photoelectron and the corresponding $\omega_{\text {surf }}$ becomes smaller. Therefore, as long as the reactance is concerned, it is speculated that the effect of the photoelectrons can be limited to the lower frequency region, which has to be examined with another simulation using the realistic parameters of photoelectrons as the future work.

\section{CONCLUSION}

To investigate the spacecraft-plasma interactions including plasma kinetic effects and EM field variation, we started to develop a numerical plasma chamber called GES by making the most use of the conventional full PIC plasma simulations. At the present stage, by using the Earth Simulator, we finished developing the core engine called NuSPACE, which is an EM PIC plasma simulation code of domain decomposition model. After intensive code tuning, we could obtain the most efficient performance in terms of vectorization and parallelization at the Earth Simulator.

In parallel to the development of NuSPACE, we have been developing a GES EM component, which handles the analysis of the antenna-plasma interactions. In this paper, we introduced the 3-D EM PIC simulations in which we particularly focused 
on the photoelectron environment around the spacecraft and antennas and analyzed the effect on the reactance of the antenna impedance. First, we confirmed the photoelectron sheath formation around the sunlit antenna as well as the satellite surface by performing the ES simulation. By using the photoelectron environment realized in the ES simulation, second, we examined the photoelectron sheath effect on the reactance part of the antenna impedance by performing the EM simulation. We could confirm the large change of the reactance below the characteristic frequency corresponding to the local plasma frequency determined by the photoelectron density. The profile of the modified reactance in the frequency domain cannot be simply explained with the past conventional theory, and we suggested the importance of electron conduction current at the solid body of antennas for the understanding of the impedance modification in the inhomogeneous plasma environment. In the current simulations, to emphasize the effect of photoelectron sheath effects, we adopted some particular conditions such as unrealistic values of energy and flux for the photoelectron emission and immobile ions. By considering these effects, the discrepancy between the simulation results described above and the previous theoretical and observational results needs to be further investigated. In addition, the usage of Gaussian-type pulse emission from the antenna feeding point for obtaining the antenna impedance might cause some artificial effects in the analysis of the antenna impedance using PIC method. This effect should be reduced by using the pulse with the amplitude as small as possible in the future works.

Although the present simulations are preliminary, we could confirm that the analysis of antenna characteristics in space plasma by using PIC simulation method including solid conducting bodies is very useful because the plasma environment can be self-consistently obtained and no assumptions are to be made for the current distribution on the antenna surface or plasma-sheath interface for inhomogeneous plasma environment in the vicinity of antenna. By advancing this simulation method and hiring more realistic parameters in terms of satellite/antenna geometry and plasma environment, we will be able to contribute to the calibration of the obtained wave data as well as the antenna design for the future mission.

\section{ACKNOWLEDGMENT}

A part of computation in this paper was performed with the KDK system of the Research Institute for Sustainable Humanosphere, Kyoto University, as a collaborative research project.

\section{REFERENCES}

[1] Frontiers in Magnetospheric Plasma Physics, ser. COSPAR Colloquia Series, vol. 16, M. Hoshino, Y. Omura, and L. J. Lanzerotti, Eds. Amsterdam, The Netherlands: Elsevier, 2005.

[2] D. E. Hastings and H. Garrett, Spacecraft Environment Interaction. Cambridge, U.K.: Cambridge Univ. Press, 1996.

[3] A. R. Martin, "A review of spacecraft/plasma interactions and effects on space systems," J. Br. Interplanet. Soc., vol. 47, no. 4, pp. 134-142, Apr. 1994

[4] E. N. Parker, "Shielding space travelers," Sci. Amer, vol. 294, no. 3, pp. 40-47, Mar. 2006.

[5] H. M. Mott-Smith and I. Langmuir, "The theory of collectors in gaseous discharges," Phys. Rev., vol. 28, no. 4, pp. 727-763, Oct. 1926.
[6] I. Langmuir and K. Blodgett, "Currents limited by space charge between concentric spheres," Phys. Rev., vol. 24, no. 1, pp. 49-59, Jul. 1924

[7] S. E. Deforest, "Spacecraft charging at synchronous orbit," J. Geophys. Res., vol. 77, no. 4, pp. 651-659, Feb. 1972.

[8] H. B. Garrett, "The charging of spacecraft surfaces," Rev. Geophys. Space. Phys., vol. 19, no. 4, pp. 577-616, Nov. 1981.

[9] H. B. Garrett and A. C. Whittlesey, "Spacecraft charging, an update," IEEE Trans. Plasma Sci., vol. 28, no. 6, pp. 2017-2028, Dec. 2000.

[10] E. C. Whipple, "Potentials of surfaces in space," Rep. Prog. Phys., vol. 44, no. 11, pp. 1197-1250, Nov. 1981

[11] P. K. Shukla and A. A. Mamum, Introduction to Dusty Plasma Physics, ser. Series on Plasma Physics. Bristol, U.K.: Inst. Phys. Pub. Inc., 2000.

[12] G. L. Delzanno, A. Bruno, G. Sorasio, and G. Lapenta, "Exact orbital motion theory of the shielding potential around an emitting, spherical body," Phys. Plasmas, vol. 12, no. 6, p. 062102, Jun. 2005.

[13] B. A. Thiébault, A. Hilgers, E. Sasot, H. Laakso, P. Escoubet, V. Génot, and J. Forest, "Potential barrier in the electrostatic sheath around a magnetospheric spacecraft," J. Geophys. Res., vol. 109, no. A12, p. A12207, Dec. 10, 2004. 10.1029/2004JA010398.

[14] M. J. Mandell, I. Katz, and D. Cooke, "Towards a more robust spacecraft charging algorithm," in Proc. 6th Spacecraft Charging Technol. Conf., 2000, pp. 251-255. AFRL-VS-TR-2001578.

[15] C. K. Birdsall and A. B. Langdon, Plasma Physics Via Computer Simulation. New York: McGraw-Hill, 1985.

[16] M. J. Mandell, I. Katz, J. M. Hilton, D. L. Cooke, and J. Minor, "Nascap2K spacecraft charging models: Algorithms and applications," in Proc. 7th Spacecraft Charging Technol. Conf., Apr. 2001, pp. 23-27.

[17] A. Hilgers, J. Forest, and J. F. Roussel. (2002, Dec). SPIS, the Spacecraft Plasma Interaction System. [Online]. Available: http://dev.spis.org/ projects/spine/home/spis

[18] J.-F. Roussel, F. Rogier, D. Volpert, G. Rousseau, J. Forest, and A. Hilgers, "Spacecraft plasma interaction software (SPIS): Numerical solvers-Methods and architecture," in Proc. 9th Spacecraft Charging Technol. Conf., 2005, Tsukuba, Japan.

[19] A. Hilgers, B. Thiébault, J.-F. Roussel, J. Forest, and E. Engwall, "Tests and validation of a new spacecraft plasma interaction software, SPIS," in Proc. 9th Spacecraft Charging Technol. Conf., 2005, Tsukuba, Japan.

[20] Y. Omura and H. Matsumoto, "KEMPO1: Technical guide to onedimensional electromagnetic particle code," in Computer Space Plasma Physics: Simulation Techniques and Softwares, H. Matsumoto and Y. Omura, Eds. Tokyo, Japan: Terra Scientific, 1993, pp. 21-65.

[21] K. S. Yee, "Numerical solution of initial boundary value problems involving Maxwell's equations in isotropic media," IEEE Trans. Antennas Propag., vol. AP-14, no. 3, pp. 302-307, Mar. 1966.

[22] R. W. Hockney and J. W. Eastwood, Computer Simulation Using Particles. New York: McGraw-Hill, 1981.

[23] I. Katz, M. J. Mandell, G. Jongeward, and M. S. Gussenhoven, "The importance of accurate secondary electron yields in modeling spacecraft charging," J. Geophys. Res., vol. 91, no. A12, pp. 13739-13744, Dec. 1986.

[24] T. Sato, "The current status of the Earth simulator," J. Earth Simul., vol. 1, no. 1, pp. 6-7, 2004

[25] M. Okada, H. Usui, Y. Omura, H. O. Ueda, K. T. Murata, and T. Sugiyama, "Application of NuSPACE to the spacecraft environment analysis," in Proc. 25th ISTS Selected Papers, 2006, in press.

[26] P. E. Glaser, "Power from the sun: Its future," Science, vol. 162, no. 857, pp. 857-861, Nov. 1968.

[27] H. Usui, H. Matsumoto, and R. Gendrin, "Numerical simulations of a three-wave coupling occurring in the ionospheric plasma," Nonlinear Process. Geophys., vol. 9, no. 1, pp. 1-10, 2002.

[28] K. G. Balmain, "The Impedance of a short dipole antenna in a magnetoplasma," IEEE Trans. Antennas Propag., vol. AP-12, no. 5, pp. 605-617, Sep. 1964.

[29] H. H. Kuehl, "Resistance of a short antenna in a warm plasma," Radio Sci., vol. 1, no. 8, pp. 971-976, 1966.

[30] M. L. Schiff, "Impedance of a short dipole antenna in a warm isotropic plasma," Radio Sci., vol. 5, no. 12, pp. 1489-1496, 1970.

[31] P. Meyer and N. Vernet, "Impedance of a short antenna in a warm magnetoplasma," Radio Sci., vol. 9, no. 3, pp. 409-416, 1974.

[32] N. Meyer-Vernet and C. Perche, "Toolkit for antennae and thermal noise near the plasma frequency," J. Geophys. Res., vol. 94, no. A3, pp. 2405$2415,1989$.

[33] K. R. Cook and B. C. Edgar, "Current distribution and impedance of a cylindrical antenna in an isotropic compressible plasma," Radio Sci., vol. 1, no. 1, pp. 13-19, 1966. 
[34] S. Adachi, T. Ishizone, and Y. Mushiake, "Transmission line theory of antenna impedance in a magnetoplasma," Radio Sci., vol. 12, no. 1, pp. 23-31, 1977.

[35] P. Meyer, N. Vernet, and P. Lassudrie-Duchesne, "Theoretical and experimental study of the effect of the sheath on an antenna immersed in a warm isotropic plasma," J. Appl. Phys., vol. 45, no. 2, pp. 700-706, Feb. 1974.

[36] M. L. Schiff and J. A. Fejer, "Impedance of antenna in a warm isotropic plasma: A comparison of different models," Radio Sci., vol. 5, no. 5, pp. 811-819, 1970.

[37] C. Béghin and E. Kolesnikova, "Surface-charge distribution approach for modeling of quasi-static electric antennas in isotropic thermal plasma," Radio Sci., vol. 33, no. 3, pp. 503-516, 1998.

[38] C. Béghin, P. M. E. Decreau, J. Pickett, D. Sundkvist, and B. Lefebvre, "Modeling of cluster's electric antennas in space: Application to plasma diagnostics," Radio Sci., vol. 40, no. 6, p. RS6008, Nov. 2005.

[39] J. J. Boonzaaier and C. W. I. Pistorius, "Thin wire dipoles-A finitedifference time-domain approach," Electron. Lett., vol. 26, no. 22, pp. 1891-1892, Oct. 1990

[40] R. Luebbers, L. Chen, T. Uno, and S. Adachi, "FDTD calculation of radiation patterns, impedance, and gain for a monopole antenna on a conducting box," IEEE Trans. Antennas Propag., vol. 40, no. 12, pp. 15771583, Dec. 1992.

[41] J. Ward, C. Swenson, and C. Furse, "The impedance of a short dipole antenna in a magnetized plasma via finite difference time domain model," IEEE Trans. Antennas Propag., vol. 53, no. 8, pp. 2711-2718, Aug. 2005.

[42] M. Tsutsui, I. Nagano, H. Kojima, K. Hashimoto, H. Matsumoto, S. Yagitani, and T. Okada, "Measurements and analysis of antenna impedance aboard the GEOTAIL spacecraft," Radio Sci., vol. 32, no. 3, pp. 1101-1126, 1997.

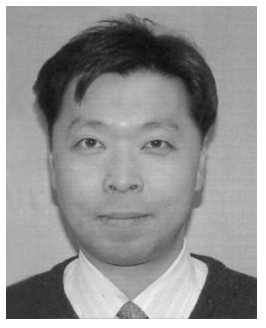

Hideyuki Usui received the B.S. degree from the Department of Electrical Engineering, Kyoto University, Kyoto, Japan, in 1986 and the M.S. and Ph.D. degrees from the Department of Electronics, Kyoto University, in 1989 and 1994, respectively.

He started as a Research Assistant with the Radio Science Center for Space and Atmosphere, Kyoto University, in 1992. He stayed as a Visiting Scientist with the Plasma Theory and Simulation Group, University of California, Berkeley, in 1997. In 1999, he was promoted to an Associate Professor with the Radio Science Center for Space and Atmosphere, Kyoto University. In 2000, the Center has been reorganized as the Research Institute for Sustainable Humanosphere, Kyoto University, where he has been currently working as an Associate Professor.

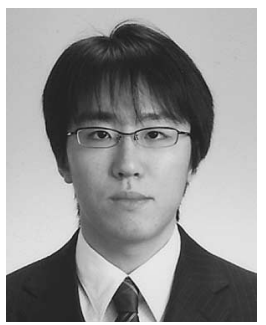

Yohei Miyake was born in Osaka, Japan, in 1982 He received the B.S. and M.S. degrees from the Department of Electrical Engineering, Kyoto University, Kyoto, Japan, in 2004 and 2006, respectively. $\mathrm{He}$ is currently working toward the Ph.D. degree at the Department of Electrical Engineering, Kyoto University.

Since 2006, he has been a Research Fellow with the Japan Society for the Promotion of Science, Tokyo, Japan. His current research interests focus on antenna analysis and design of electric field sensor in space plasma by using numerical simulation.

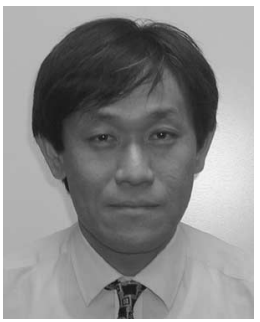

Masaki Okada received the B.S. degree from the Department of Electrical Engineering, Kyoto University, Kyoto, Japan in 1988 and the M.S. and Ph.D. degrees from the Department of Electronics, Kyoto University, in 1990 and 1994, respectively.

He been a Research Assistant with the National Institute of Polar Research, Itabashi, Japan, since 1994

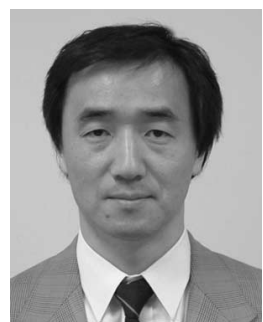

Yoshiharu Omura received the B.S., M.S., and $\mathrm{Ph} . \mathrm{D}$. degrees from the Department of Electronics and Electrical Engineering, Kyoto University, Kyoto, Japan, in 1980, 1982, and 1985, respectively.

$\mathrm{He}$ started as a Research Assistant with the Department of Electrical Engineering, Kyoto University, in 1985. He stayed as a National Research Council Research Associate with Goddard Space Flight Center in 1991-1992. In 2000, he was promoted to a Professor with the Radio Science Center for Space and Atmosphere, Kyoto University, which was reorganized as the Research Institute for Sustainable Humanosphere, Kyoto University, in 2004. He has been working as a Professor with the Laboratory of Computer Simulation for Humanospheric Sciences.

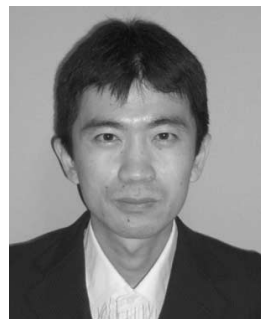

Tooru Sugiyama received the B.S. degree from the Department of Science, Nagoya University, Nagoya, Japan, in 1993 and the M.S. and Ph.D. degrees from the Department of Science, University of Tokyo, Tokyo, Japan, in 1995 and 1998, respectively.

He started as a Research Fellow with the Japan Society for the Promotion of Science, Tokyo Institute of Technology, and Max-Planck-Institut and as a Center of Excellence Researcher with the Radio Science Center for Space and Atmosphere, Kyoto University, Kyoto, Japan. He has been a Research Scientist with the Earth Simulator Center, Japan Agency for Marine-Earth Science and Technology, Kanazawa, Japan.

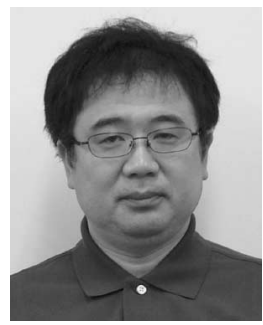

Ken T. Murata received the B.S. degree from the Department of Mechanical Engineering, Kyoto University, Kyoto, Japan, in 1988 and the M.S. and Ph.D. degrees from the Department of Electronics, Kyoto University, in 1992 and 1995, respectively.

He started as a Research Assistant with the Department of Computer Science, Ehime University, Matsuyama, Japan, in 1995, where he was engaged in studies concerning numerical calculation technique and its application to space physics. In 2003, he moved to the Center for Information Technology, Ehime University, as an Associate Professor. So far, he has been working in visualization and virtual reality of computer simulations of space plasma and network database for spacecraft and ground-based observations.

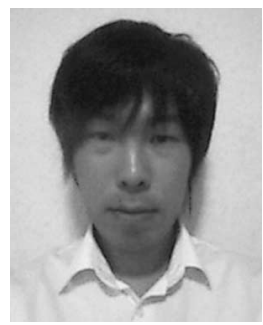

Daisuke Matsuoka received the B.S. and M.S. degrees from the Department of Computer Science, Ehime University, Matsuyama, Japan, in 2003 and 2005 , respectively. He is currently working toward the Ph.D. degree with the Graduate School of Science and Engineering, Ehime University.

His research interests are 3-D visualization analysis and virtual reality for space plasma simulation.

Mr. Matsuoka is a member of the Society of Geomagnetism and Earth, Planetary and Space Sciences and the Volcanological Society of Japan.

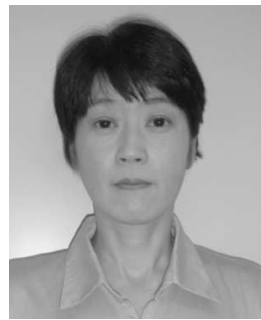

Hiroko O. Ueda received the Ph.D. degree from the Department of Electronic Engineering, University of Electro-Communications, Tokyo, Japan, in 1994.

$\mathrm{He}$ is an Associate Senior Researcher with Japan Aerospace Exploration Agency, Tsukuba, Japan, since 2001. She was a Research Assistant with Chiba University, Chiba, Japan, from 1992 to 2001. 